\title{
Oligomerization driven by phase separation
}

\author{
I. Myrgorodska, ${ }^{[a]}$ I. Colomer ${ }^{[a]}$ and S.P. Fletcher ${ }^{*[a]}$
}

\begin{abstract}
Chemical reactions occurring within liquid-liquid phase separated systems drive numerous biological processes and have likely been involved in the emergence of first cellular compartments. Here we report a reaction whose product distribution is drastically affected by the presence of a liquid/liquid interface. Under phase separated conditions, the system produces a dynamic library of increasingly complex oligomeric structures. This reactivity is in sharp contrast to results under homogeneous conditions; when there is no phase separation only two thermodynamically stable products are formed. The reactivity observed using phase separated conditions stems from generating a dynamic out-of-equilibrium self-assembling system. This work shows that relatively complex oligomeric products that would not be otherwise easily accessible can be made by biphasic out-ofequilibrium processes.
\end{abstract}

\section{Introduction}

Liquid-liquid phase separation lies at the core of many biological processes. For instance, it allows components to rapidly concentrate, facilitating spatiotemporal regulation of cellular functions. ${ }^{[1,2]}$ Membranelles organelles such as centromeres, $\mathrm{P}$ bodies, and nucleolus ${ }^{[3,4]}$ are dense liquid droplets which regulate nucleation of actin filaments, microtube formation, transcription, splicing and cellular signalling (Figure 1a). Aside from known biology, assembly of material at the liquid-liquid interface may also have been important to the origin of cellular compartmentalisation. ${ }^{5,6]}$ Understanding reactions occurring at the interface of two liquid phases is therefore of great importance.

Phase separation can limit the effective concentration of reaction partners and modify reaction kinetics vs homogeneous reactions. Some organic reaction rates benefit from 'on water' reaction conditions ${ }^{[7]}$ which effectively concentrates hydrophobic components by addition of water. In rationally designed biphasic systems, hydrophobic molecules can react with hydrophilic components across the interface, generating amphiphilic species. Self-assembly of the

[a] Dr. I. Myrgorodska, Dr. I. Colomer, Prof. S. P. Fletcher University of Oxford

Chemistry Research Laboratory

12 Mansfield Road

Oxford OX1 3TA

E-mail: stephen.fletcher@chem.ox.ac.uk

Supporting information for this article is given via a link at the end of the document. amphiphilic products of such interfacial reactions has been used in models for primitive membranes. ${ }^{[8-12]}$ Combining selfassembly processes with a concurrent destruction mechanism represents one of the current challenges in the field of systems chemistry. ${ }^{[13,14]}$.

How liquid-liquid phase separation can control reaction products is well understood in reversible reactions under thermodynamic control, which can be driven by removing reaction products from the medium. The effect of phase separation on product distribution in dynamic combinatorial libraries (DCLs) has been investigated. ${ }^{[15,16]}$ Hafezi and Lehn ${ }^{[17]}$ studied a DCL which undergoes constitutional reorganization under reversible phase separation and several reports indicate that two phase systems increase the scope and possibilities of DCLs. ${ }^{[18,19]}$

While changes in the product distribution of equilibrating reversible systems is well documented, there are few reports of phase separation driving systems away from equilibrium, opening new bond destruction or formation pathways and initiating new modes of product selection. ${ }^{[20]}$ One case involves olefin metathesis between hydrophobic and hydrophilic alkenes. In this reaction, phase separation enables a secondary metathesis reaction which consumes the newly formed surfactant alkene (Figure 1b). ${ }^{[11]} A$ second example involves the recent beautiful demonstration of using liquid phase-separation as a mechanism for selecting products from a library of interacting molecules (Figure 1c). ${ }^{[22]}$ In both examples resupply of starting material is required to maintain the systems out-of-equilibrium.

In our previous work (Figure 1b) ${ }^{[21]}$, alkene metathesis between phase separated reactants generated non-thermodynamically stable transient species. Here we sought to study a more elaborate system that could generate a diverse population of products. Two competing hydrophilic starting materials were used, one with a single reactive site, the other with two handles for reaction. This design would allow formation of several possible transient intermediates and thermodynamic products (Figure 1d). Drastically different products were observed under different conditions; homogeneous reactions gave two crossmetathesis products, while under biphasic conditions a diverse array of complex oligomers was observed. In this communication, we characterize these products, the kinetics of these reactions and demonstrate that phase separation can play a crucial and unusual role in greatly increasing complexity of a chemical system by making it both out-of-equilibrium and oligomer-producing. 
a. Liquid-liquid phase separation in biology

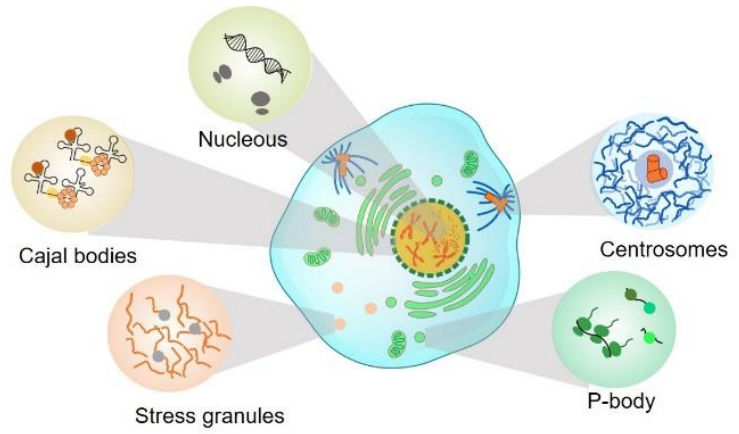

b. Transient replicator triggered by phase separation ${ }^{[21]}$

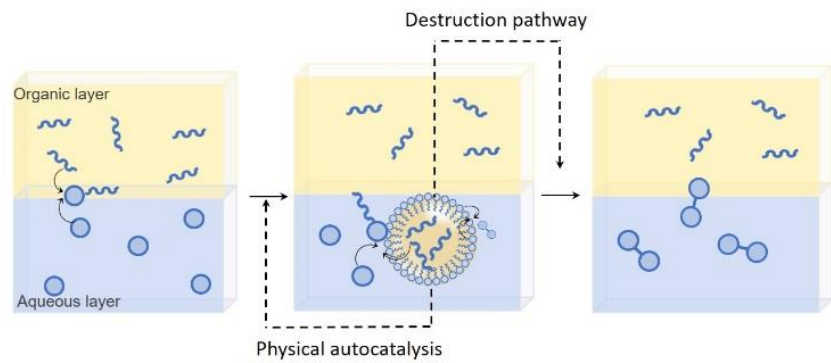

c. Selection by phase separation ${ }^{[22]}$

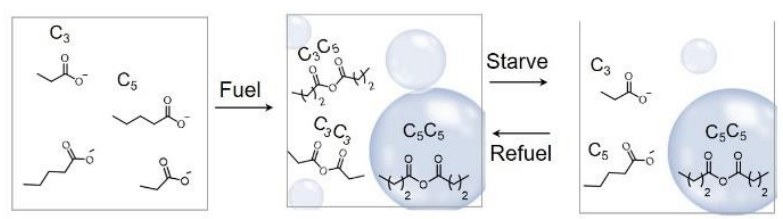

d. This work

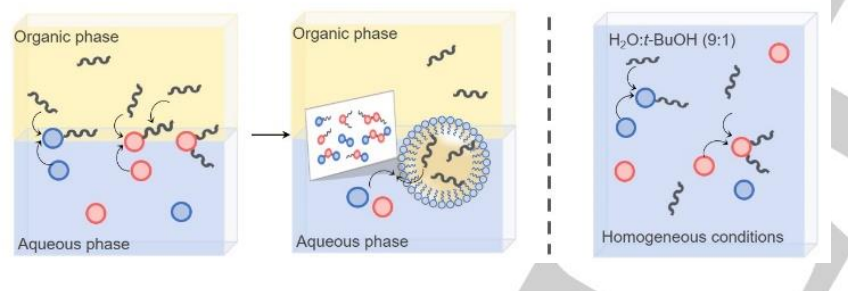

Figure 1. The importance of phase separation and liquid/liquid interfaces. a) Schematic representation of membrane-less organelles formed by liquid-liquid phase separation in eukaryotic cells. b) Transient self-assembling replicators based on biphasic olefine cross-metathesis. c) Selection of nonequilibrium phaseseparated product by a persistency mechanism. ${ }^{[22]}$ d) This work: complex oligomerization driven by phase separation. Under homogenous conditions only two products are observed.

\section{Results and Discussion}

Previously a transient replicator was generated from hydrophobic 2 and hydrophilic 1a with Grubbs $2^{\text {nd }}$ generation catalyst under biphasic conditions, where cross-metathesis first formed amphiphilic 3a. Kinetic studies demonstrated the formation of $\mathbf{3 a}$ has a sigmoidal rate profile, characteristic of an autocatalytic process. ${ }^{[21,23]}$ After reaching the critical micellar concentration $\mathbf{3 a}$ self-assembles into micelles, and subsequently gets destroyed in a second metathesis reaction to form what must be the thermodynamic product under the reaction conditions.

Here we used two hydrophilic components (1a and 1b) with hydrophobic 2 . Initially, we examined metathesis reaction under homogeneous conditions, where 1a, 1b, Grubbs II catalyst and excess 2 were all dissolved in a mixture of $t$ $\mathrm{BuOH}-\mathrm{D}_{2} \mathrm{O}$ (9:1 volume ratio). Fast conversion (Figure 2) to products $\mathbf{3 a}$ and $\mathbf{3 c}$ was observed, and both molecules were shown to be stable under these conditions. Single chain metathesis product $\mathbf{3 b}$ was not observed under these conditions due to the fast rate of second metathesis reaction and the limited resolution our analysis method. Interestingly, a similar experiment (using a mixture of $t-\mathrm{BuOH}-\mathrm{D}_{2} \mathrm{O}$ ) without hydrophobic 2 (only using $\mathbf{1 a}$ and $\mathbf{1 b}$ ) showed only limited alkene cross-metathesis, with low conversion even after $22 \mathrm{~h}$ (see SI Fig. S3). Slow homodimerization under homogeneous conditions is expected for styrene derivatives which are type II olefins using Grubbs II catalyst, and this observed behaviour is consistent with previous olefin cross-metathesis studies. ${ }^{[24,25]}$

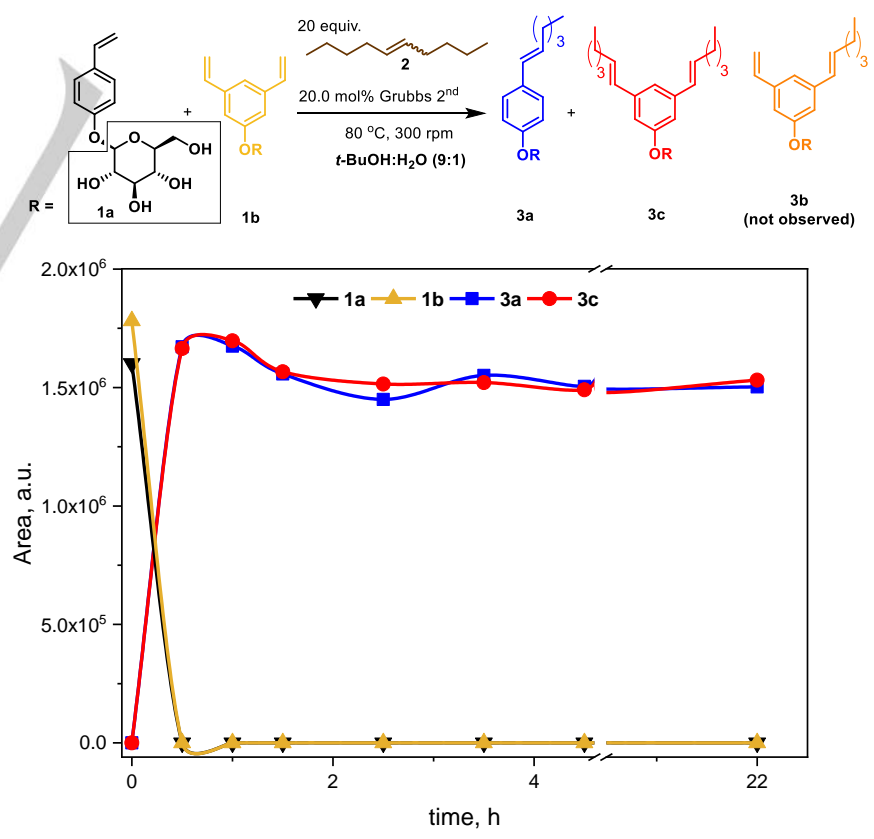

Figure 2. Alkene cross-metathesis between hydrophobic alkene 2 and hydrophilic olefins 1a (black) and 1b (yellow) under homogeneous conditions $t-\mathrm{BuOH}-\mathrm{D}_{2} \mathrm{O}$ (9:1 volume ratio) 

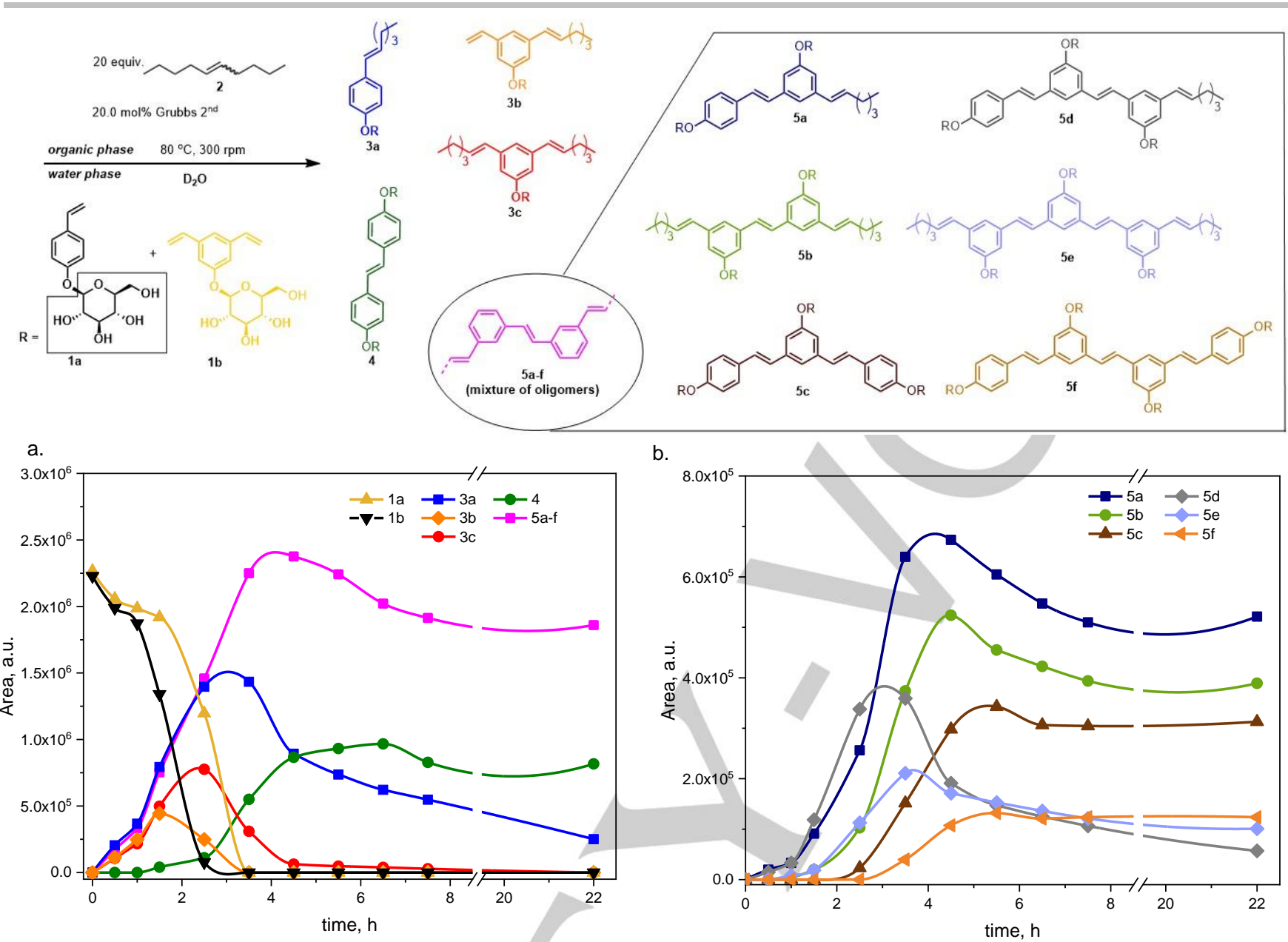

Figure 3. Phase separated alkene metathesis reaction using two different hydrophilic alkenes $\mathbf{1 a}$ and $\mathbf{1 b}$ with hydrophobic alkene $\mathbf{2}$ results in: a) the transient formation of species $\mathbf{3 a}, \mathbf{3 b}$, and $\mathbf{3 c}$ that are further converted into thermodynamic product $\mathbf{4}$ and a complex mixture of oligomers $\mathbf{5 a - f}$. This mixture of oligomers has been carefully examined with a composition formed by: $\mathbf{b})$ two species incorporating two monomers $\mathbf{5} \mathbf{a}$ and $\mathbf{5} \mathbf{b}$, three species integrating three monomeric units $5 \mathrm{c}, 5 \mathrm{~d}$ and $\mathbf{5 e}$ and one product formed by four monomers $5 \mathrm{f}$.

Phase separated conditions were achieved by hydrophobic alkene 2 forming a discrete layer on top of an aqueous phase containing an equimolar mixture of $\mathbf{1 a}$ and $\mathbf{1 b}$. Reaction between hydrophilic $\mathbf{1 a}$ and $\mathbf{1 b}$ and hydrophobic $\mathbf{2}$ first resulted in the formation of amphiphilic alkenes $\mathbf{3 a}, \mathbf{3 b}$ and $\mathbf{3 c}$ (blue, orange and red line in Figure $3 \mathrm{a}$ ). These products then react further to give more complex oligomeric structures (Figure 3b). In agreement with our previous study, 3a suffers a second alkene metathesis reaction leading to the destruction surfactant $\mathbf{3} \mathbf{a}$ and formation of water-soluble thermodynamic product 4 (green line in Figure 3a). Previously reported mechanistic investigation strongly suggest that second metathesis step is occurring at the interface between aqueous and organic phase. ${ }^{[21]}$ Transient metastable species $\mathbf{3 b}$ and $\mathbf{3 c}$ also take part in further alkene metathesis reactions to form diverse molecular architectures 5a-f (purple line in Figure 3a, for the combined concentration).

In order to identify the newly generated species, products 5a-f were individually isolated using preparative HPLC. Careful characterization of the isolated compounds, structural assignments, and analysis of their kinetic profiles allowed us to propose a reaction pathway to explain the formation of the increasingly complex oligomers (see SI for the complete analysis details).

The reaction starts with formation of the two transient surfactants species $\mathbf{3 a}$ (from $\mathbf{1 a}$ ) and $\mathbf{3 b}$ (from $\mathbf{1 b}$, via a single metathesis with $\mathbf{2}$ ). $\mathbf{3 b}$ can undergo a second alkene metathesis reaction to give $\mathbf{3 c}$, and $\mathbf{3} \mathbf{c}$ can then react, either with $\mathbf{3 a}$ or with another molecule of $\mathbf{3 c}$ to give $\mathbf{5 a}$ or $\mathbf{5 b}$ respectively. Both $\mathbf{5 a}$ and $\mathbf{5 b}$ can further react with $\mathbf{3 a}$ and $\mathbf{3 c}$ leading to $\mathbf{5 d}, \mathbf{5 e}$ and $\mathbf{5 c}$ (Figure 4). In this stepwise oligomerization reaction, we have detected products formed from up to four monomers, $5 \mathbf{h}$. As populations of molecules grow and deplete, some structures appear to be more persistent than others. (Figure 3b).

Self-assembly of $\mathbf{3 a}$ into micelles has been confirmed by dynamic light scattering (DLS) of pure 3a and images taken by transmittance electron microscope (TEM). The micelle size is $\sim 27 \pm 9 \mathrm{~nm}$. A fluorometric assay with 1,6-diphenyl-1,3,5hexatriene (DPH) as fluorescent dye has been used to establish critical micellar concentration $(\mathrm{cmc})$. The $\mathrm{cmc}$ of $\mathbf{3 a}$ was determined to be $\sim 0.7 \mathrm{mM}$ (see SI section 5 for further details). 


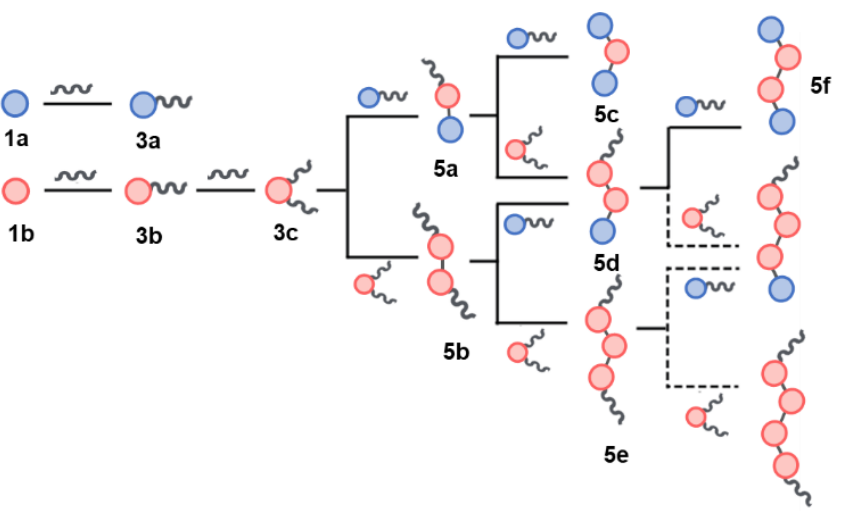

Figure 4. a) Schematic tree depicting possible pathways showing the formation of increasingly complex structures $\mathbf{5 a - f}$, from hydrophilic components $\mathbf{1 a}$ and $\mathbf{1 b}$ and hydrophobic alkene;.

To support our assumption that self-assembly of the first metathesis product $\mathbf{3 a}$ facilitates additional metathesis steps and drives product diversity we conducted similar biphasic reactions with hydrophobic alkene trans-3-hexene, which has a shorter chain. The corresponding metathesis product of $\mathbf{1 a}$ and trans-3-hexene does not self-assemble in water (no aggregates detected by DLS). Kinetic analysis of the system showed that only small amounts of thermodynamic and oligomeric products are formed (SI: Figure S4). On the other hand, the addition of Triton- $X$, a neutral surfactant, to the biphasic system comprising of $\mathbf{1 a}, \mathbf{1 b}$ and $\mathbf{2}$ facilitates the reaction and removes the lag period associated with the absence of micelles at the start of the reaction (SI: Figure S5). These experiments support the idea that the interface of plays a crucial role in promoting oligomerisation.

It is important to note that we conducted a biphasic reaction using only $\mathbf{1 b}$ as hydrophilic alkene with $\mathbf{2}$ and to our surprise, only limited amount of product $\mathbf{3 c}$ was detected by UPLC (SI: Figure S3), and no other oligomerization products were observed. Further investigations revealed that unlike $\mathbf{3 a}$, product $\mathbf{3 c}$ is not a surfactant and does not self-assemble under the reaction conditions. Emphasising that micelles are essential for the reaction to be dynamic, as they lower the interfacial tension and allow the reactive species and the catalyst to be in close proximity. This will also promote subsequent reactions driving the oligomerisation process. Thus, a biphasic system that does not involve self-assembly products is likely not to generate complex molecular architectures.

\section{Conclusions}

In conclusion, we have shown how phase-separation can be the origin of altered chemical reactivity, triggering complex phenomena that does not ocurr when there is no liquid-liquid interphase. Here phase separation results in a tremendous increase in molecular complexity, and a fairly dynamic population of species emerges.

Reactions which assemble monomers into polymeric chains are central to biology. In the absence of specific and powerful catalysts (i.e. enzymes), oligomerization processes are generally not favoured. Nature overcomes thermodynamic and kinetic limitations ${ }^{[26]}$ in oligomerization reactions by operating under out-of-equilibrium conditions. Therefore, identifying new pathways facilitating oligomerisation process could provide insight into the basic principles of life's selforganisation. ${ }^{[27,28,29]}$

\section{Experimental Section}

General experimental details. Reagents obtained from SigmaAldrich, Alfa, Fluorochem and $\mathrm{TCl}$ suppliers were used directly as supplied. All anhydrous reactions were carried out in flame-dried glassware and under an inert atmosphere of argon provided by a balloon. All reactions were stirred with magnetic followers. Flash column chromatography was performed using silica gel ( $60 \AA$, 0.033 $0.070 \mathrm{~mm}, \mathrm{BDH})$. TLC analyses were performed on Merck Kiesegel 60 F254 $0.25 \mathrm{~mm}$ precoated silica plates. See supplementary information for synthetic procedures.

General procedure for biphasic Ru-catalysed alkene crossmetathesis. To a flame-dried flask, charged with a catalytic amount of $\mathrm{Ru}$-catalyst, under $\mathrm{Ar}$, at room temperature, was added a solution of hydrophilic alkene in dry $\mathrm{D}_{2} \mathrm{O}$ (previously degassed, bubbling Ar during 30 min.). Hydrophobic alkene was added and the mixture was stirred at the appropriate temperature and stirring speed. The reaction was monitored using Ultra Performance Liquid Chromatography (UPLC) until completion. The solvent was evaporated under reduced pressure to give the corresponding product that was purified by chromatography on silica gel using the appropriate mixture of eluents or by semipreparative high performance liquid chromatography (HPLC) connected to automatic collection unit.

Kinetic analysis. Kinetic analyses were performed using a Waters ACQUITY ultra-performance liquid chromatography (UPLC) $\mathrm{H}$-Class system with photodiode array (PDA) detector. Instrument control and data processing were performed using Empower software. ACQUITY UPLC BEH C18 column, $2.1 \times 50 \mathrm{~mm}$ with a $1.7 \mu \mathrm{m}$ size particle was used. A mixture of $\mathrm{ACN}: \mathrm{H}_{2} \mathrm{O}$ with a gradient of $5: 95 \rightarrow 95: 5$ over 6 min was used as mobile phase. See supplementary information for more details, including chromatograms (Supplementary Figures 12-13).

Compounds characterisation. ${ }^{1} \mathrm{H}$ NMR and ${ }^{13} \mathrm{C}$ NMR spectra were recorded on a $400 \mathrm{MHz}, 500 \mathrm{MHz}$ or $600 \mathrm{MHz}$ spectrometers in $\mathrm{CDCl}_{3}$ or $\mathrm{CD}_{3} \mathrm{OD}$ and referenced to residual solvent peaks (Supplementary Figures 22-31). Infrared spectra were recorded as thin films of neat samples on a Bruker Tensor 27 FT-IR spectrometer equipped with Attenuated Total Reflectance sampling accessories. High-resolution mass spectra are given to four decimal places and were recorded on a Bruker MicroTof (resolution $=10,000$ FWHM) under conditions of electrospray ionisation (ESI), electronic ionisation (EI) or chemical ionisation $(\mathrm{Cl})$. Optical rotations were measured at $25^{\circ} \mathrm{C}$ using a sodium lamp in the appropriate solvent. Melting points (m.p.) were obtained from recrystallised samples using a Lecia VMTG heatedstage microscope and are uncorrected.

\section{Acknowledgements}

We thank the European Research Council (Consolidator Grant 'autocat', 681491) for funding.

Keywords: autocatalysis $\cdot$ phase separation • alkene metathesis $\cdot$ systems chemistry

[1] A. A. Hyman, C. A. Weber, F. Jülicher, Annu. Rev. Cell Dev. Biol. 2014, 30, 39-58.

[2] S. Alberti, Curr. Biol. 2017, 27, R1097-R1102.

[3] S. F. Banani, H. O. Lee, A. A. Hyman, M. K. Rosen, Nat. Rev. Mol. Cell Biol. 2017, 18, 285.

[4] S. Elbaum-Garfinkle, Y. Kim, K. Szczepaniak, C. C.-H. Chen, C. R. Eckmann, S. Myong, C. P. Brangwynne, Proc. Natl. Acad. Sci. U.S.A. 2015, 112, 7189-7194.

[5] C. D. Keating, Acc. Chem. Res. 2012, 45, 2114-2124. 
[6] A. Pohorille, D. Deamer, Microbiol. Res. 2009, 160, 449456.

[7] S. Narayan, J. Muldoon, M. G. Finn, V. V. Fokin, H. C. Kolb, K. B. Sharpless, Angew. Chem. Int. Ed. 2005, 44, 32753279.

[8] P. A. Bachmann, P. L. Luisi, J. Lang, Nature 1992, 357, 5759.

[9] P. L. Luisi, P. Stano, The minimal cell: the biophysics of cell compartment and the origin of cell functionality 2010, Springer Science \& Business Media.

[10] A. J. Bissette, , B. Odell, S. P. Fletcher, Nat. Commun. 2014, 5, 4607.

[11] E. A. J. Post, A. J. Bissette, S. P. Fletcher, Chem. Commun. 2018, 54, 8777-8780.

[12] E. A. J. Post, S. P. Fletcher, J. Org. Chem. 2019, 84, 27412755.

[13] G. Ashkenasy, T. M. Hermans, S. Otto, A. F. Taylor, Chem. Soc. Rev. 2017,46, 2543-2554

[14] O. Š Miljanić, Chem 2017, 2, 502--524.

[15] J. Li, P. Nowak, S. Otto, J. Am. Chem. Soc. 2013, 35, 92229239.

[16] R. A. Hunt, S. Otto, Chem. Comm. 2011, 47, 847-858.

[17] N. Hafezi, J. M. Lehn, J. M., Am. Chem. Soc. 2012, 134, 12861-12868.

[18] R. Pérez-Fernández, M. Pittelkow, A. M. Belenguer, J. K. Sanders, Chem. Comm. 2008, 15, 1738-1740.

[19] F. M. Mansfeld, H. Y. Au-Yeung, J. K. Sanders, S. Otto, J. Sys. Chem. 2010, 1,12.

[20] S. M. Morrow, I. Colomer, S. P. Fletcher, Nat. Commun. 2019 10, 1011.

[21] I. Colomer, S. M. Morrow, S. P. Fletcher, Nat. Commun. 2018 9, 2239.

[22] M. Tena-Solsona, C. Wanzke, B. Riess, A. R. Bausch, J. Boekhoven, Nat. Commun. 2018, 9, 2044.

[23] A. J. Bissette, S. P. Fletcher, Angew. Chem. Int. Ed. 2013, 49, 12800-12826.

[24] W. E. Crowe, Z. J. Zhang, J. Am. Chem. Soc. 1993, 115, 10998-10999.

[25] A. K. Chatterjee, T.-L. Choi, D. P. Sanders, R. H. Grubbs, J. Am. Chem. Soc. 2003, 125, 11360-11370.

[26] E. Schrödinger, What is Life? The Physical Aspect of the Living Cell 1944, Cambridge Univ. Press.

[27] M. Morasch, J. Liu, C. F. Dirscherl, A. laneselli, A. Kühnlein, K. Le Vay, P. Schwintek, S. Islam, M. K. Corpinot, B. Scheu, D. B. Dingwell, Nature Chem. 2019, 11, 779-788.

[28] P. Canavelli, S. Islam, M. W. Powner, Nature 2019, 571, 546-549.

[29] I. Colomer, A. Borissov, S. P. Fletcher, Nat. Commun. 2020, 11, 176. 
WILEY-VCH

\section{ARTICLE}

\section{ARTICLE}

The more the merrier: Phase separation triggers the stepwise oligomerization of two building blocks generating a library of dynamic species, leading to a structurally diverse, complex mixture of molecules. In contrast only two thermodynamically stable products are obtained with no phase separation.

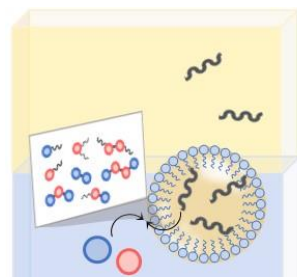

I. Myrgorodska, I. Colomer, S.P. Fletcher $^{*}$

Page No. - Page No.

Oligomerization driven by phase separation 